\title{
Reservoir Wave Paradigm: An Implementation and Sensitivity Analysis
}

\author{
Markus Ebner $^{1}$, Bernhard Hametner ${ }^{1,2 *}$, Stefanie Parragh ${ }^{1,2}$, Siegfried Wassertheurer ${ }^{2}$ \\ ${ }^{1}$ Department of Analysis and Scientific Computing, Vienna University of Technology, Wiedner Hauptstraße 8-10, \\ 1040 Vienna, Austria \\ ${ }^{2}$ Health and Environment Department, AIT Austrian Institute of Technology GmbH, Donau-City-Straße 1, 1220 \\ Vienna, Austria; *bernhard.hametner@ait.ac.at
}

Simulation Notes Europe SNE 25(3-4), 151 - 156

DOI: $10.11128 /$ sne.25.tn. 10307

Received: March 11, 2015; Revised September 25, 2015;

Accepted: October 11, 2015

Abstract. Striving for a better understanding of complex phenomena of hemodynamics and the need of reliable, clinically relevant information on the circulatory system are the driving forces to refine already existing cardiovascular models. A recent modelling theory is called the reservoir wave paradigm (RWP) and merges two existing models in order to address two important phenomena at the same time: arterial compliance and wave propagation. The aim of this article is to present its inherent concept and to illustrate its sensitivity to different model parameters. For this purpose the RWP is applied to an exemplary pressure waveform originating at the ascending aorta by using two different estimators of systolic time duration as well as two different choices of minimization intervals for the subsequent reservoir pressure fitting. The findings indicate a substantial impact of the chosen optimization interval. In contrast, the effect of the regarded estimators of systolic time duration on the waveform might be considered as negligible.

\section{Introduction}

Cardiovascular modelling plays a key role in order to quantify the physical state of the human circulatory system. In this context, the investigation of time and location dependent waveforms of arterial pressure and flow are of particular interest, since they are capable to provide indicators for cardiovascular diseases. For example arterial stiffness and wave reflection parameters have been revealed as clinically relevant predictors of risk and have been suggested to be incorporated into clinical practice [1-5].
To date various model concepts for describing the pressure and flow waveforms have been proposed. Beside the frequently used approaches of wave analysis, wave separation or the Windkessel method, a recently emerged theory is called the reservoir wave paradigm (RWP). Whereas the former methods concentrate on either the wave behaviour or the Windkessel behaviour, the RWP aims to incorporate both phenomena by separating the measured pressure waveform in an arterial compliance-related reservoir pressure and a waveassociated excess pressure [6]. At present the justification of this fairly new approach and its inherent assumptions are highly debated [7,8]. However, in this article it shall be focussed on the underlying model concept of the RWP and its sensitivity to the model parameters of systolic time duration and optimization intervals necessary to obtain the reservoir pressure.

The pressure separation according to the RWP can be performed in various ways depending on the availability of pressure and flow waveforms. In this work, the commonly used method of only using pressure curves is derived and applied to a pressure waveform originating at the ascending aorta. The underlying concept of fitting the reservoir pressure waveform to the measured one in later diastole requires an estimation of systolic time duration and a corresponding appropriate minimization interval. For this purpose, two different estimators of systolic time durations are presented and used to define two different optimization intervals. All methods are implemented in MATLAB and described step-by-step. Based on the results of this implementation, the impact of the chosen algorithm parameterization is discussed and analysed. 


\section{Methods}

\subsection{Reservoir Theory}

The RWP is based on the assumption that the actual pressure and flow waveforms are shaped by two prevailing phenomena within the arterial tree: the arterial compliance and wave propagation related phenomena [9].

The former describes the 'reservoir effect' of cushioning and recoiling of the major arteries, which is mainly determined by the ascending aorta as the most elastic part within the arterial tree [10]. This phenomenon is modelled by a classic two-element Windkessel concept [11].

The latter takes account for propagation phenomena of travelling waves produced by every heartbeat. Due to reflections, caused by impedance mismatches along the arterial tree, the actual waveforms can be regarded as superposition of forward and backward travelling waves. This phenomenon depends strongly on local properties.

Separation of pressure. According to the RWP, a reasonable ansatz is now to divide the actual pressure waveform $p$ along the arterial tree into an instantaneous sum of a time-dependent arterial compliance-related reservoir pressure $p_{\text {res }}$ and the remaining local, waveassociated pressure $p_{\text {ex }}$ depending on time $t$ and distance $x$ along the arteries [6], i.e.

$$
p(x, t)=p_{\text {res }}(t)+p_{\text {ex }}(x, t) .
$$

Hence, the compliance-related reservoir pressure is implicitly regarded as spatially uniform but shifted in time that it takes the waveform from the ascending aorta to arrive at the regarded vessel. Therefore, the observable differences in pressure waveforms along the arterial tree shall be fully described by the local excess pressure according to the RWP [12].

The actual pressure separation (1) is performed by computing the reservoir pressure $p_{\text {res }}$ from a given pressure waveform $p$ which then defines the remaining excess pressure $p_{\text {ex }}$.

Reservoir pressure based on flow. The reservoir effect is related to the change of volume in time within the arterial tree and leads to the classic two-element Windkessel equation

$$
\frac{d\left(p_{\text {res }}-P_{\infty}\right)}{d t}(t)=\frac{p_{\text {res }}(t)-P_{\infty}}{R C}-\frac{q_{\text {in }}(t)}{C}
$$

where $P_{\infty}$ denotes the asymptotic pressure, $R$ the arterial resistance, $C$ the arterial compliance and $q_{\text {in }}$ the flow into the arterial tree right after the left ventricle [6]. It shall be assumed that $0 \leq t \leq T_{b}$ describes one heartbeat with time duration $T_{b}$ from the beginning of systole $(t=0)$ to the end of diastole $\left(t=T_{b}\right)$. The time $t=T_{s}$ with $0<T_{s}<T_{b}$ refers to the closure of the aortic valve and shall indicate the transition from systole to diastole. From this time the flow $q_{\text {in }}$ is approximately zero and the wave activity decreases, leading to a negligible excess pressure at the end of diastole. Thus, for given parameters $R, C$ and $P_{\infty}$ the unique solution of (2) is defined by the initial value $p_{\text {res }}(0)=p(0)$ and reads

$$
\begin{aligned}
p_{\text {res }}(t)= & \frac{e^{-t /(R C)}}{C} \int_{0}^{t} q_{\text {in }}(s) e^{s /(R C)} d s \\
& +\left(p(0)-P_{\infty}\right) e^{-t /(R C)}+P_{\infty}
\end{aligned}
$$

for $0 \leq t \leq T_{b}$. By assuming the ventricular flow $q_{i n}$ to be zero during diastole, the solution is given by

$$
p_{\text {res }}(t)=\left(p_{\text {res }}\left(T_{s}\right)-P_{\infty}\right) e^{-\left(t-T_{s}\right) /(R C)}+P_{\infty}
$$

for $T_{s} \leq t \leq T_{b}$. Hence, provided that a simultaneously measured flow $q_{i n}$ and an estimation of $T_{s}$ are available, one might fit the reservoir pressure (4) to the measured pressure $p$ in diastole in order to get approximations on the asymptotic pressure $P_{\infty}$ and the time constant $\tau:=R C$ in the first place. A subsequent minimization of the difference between the reservoir pressures (3) and (4) at $t=T_{s}$ yields the remaining parameters of $R$ and $C$. Consequently, the reservoir pressure $p_{\text {res }}(t)$ is fully described by (3) for all $0 \leq t \leq T_{b}$.

The procedure described above was essentially done in [6], which revealed an excess pressure approximately proportional to the flow in the ascending aorta, i.e.

$$
q_{\text {in }}=\zeta p_{\text {ex }}=\zeta\left(p-p_{\text {res }}\right)
$$

with $\zeta>0$. The proportionality (5) basically states Ohm's law in hydraulic analogy and was one of the key findings of the RWP. In further consequence, the observation (5) became a crucial assumption and led to the elimination of the constraining requirement of flow knowledge.

Reservoir pressure without flow. By assuming the relationship (5) and inserting it into (2) one obtains the ordinary differential equation

$$
\begin{gathered}
\frac{d\left(p_{\text {res }}-P_{\infty}\right)}{d t}(t)+(a+b)\left(p_{\text {res }}(t)-P_{\infty}\right) \\
=a\left(p(t)-P_{\infty}\right)
\end{gathered}
$$


with the rate constants $a=\zeta / C$ and $b=1 /(R C)$ at the ascending aorta. Both rate constants have the same SIunit: $1 /$ s. Similarly to (3), the solution of (6) reads

$$
\begin{array}{r}
p_{\text {res }}(t)=e^{-(a+b) t} \int_{0}^{t} a\left(p(s)-P_{\infty}\right) e^{(a+b) s} d s \\
+\left(p(0)-P_{\infty}\right) e^{-(a+b) t}+P_{\infty}
\end{array}
$$

for $0 \leq t \leq T_{b}$ whereby the approximated exponential decay (4) in diastole corresponds to

$$
p_{\text {res }}(t)=\left(p_{\text {res }}\left(T_{s}\right)-P_{\infty}\right) e^{-b\left(t-T_{S}\right)}+P_{\infty}
$$

in the new variables for $T_{s} \leq t \leq T_{b}$.

In the following the pressure separation (1) via (7) and ( 8 ) as described above shall be implemented. Moreover, two different estimations of the time duration of systole $T_{S}$ by the mere knowledge of the pressure waveform will be presented.

\subsection{Data}

The investigated pressure curve is taken from [13], which in turn references to [14], and illustrates a pressure waveform of one heart cycle at the ascending aorta. It is given in terms of the arrays of pressure $p$ (in unit $\mathrm{mmHg}$ ) and time $t$ (in s). The sampling rate of the pressure curve is $0.0078 \mathrm{~s}$, which corresponds to a sampling frequency of $128 \mathrm{~Hz}$. Due to the digitized nature of the data, all operations shall be understood as discrete ones in the following.

\subsection{Duration of systole}

Two estimation methods of the duration of systole are used in this article. The first is based on the maximum curvature of the pressure $p$ and is computed by

$$
T_{s, c}:=\underset{t^{\prime}}{\operatorname{argmax}}\left(\frac{d^{2} p}{d t^{2}}\left(t^{\prime}\right)\right)
$$

with $t^{\prime}$ being within a range around an initial guess of $T_{S}$.

The second method relies on the minimum derivation of the pressure $p$ and is defined by

$$
T_{s, d}:=\underset{t^{\prime}}{\operatorname{argmin}}\left(\frac{d p}{d t}\left(t^{\prime}\right)\right)
$$

with $0 \leq t^{\prime} \leq T_{b}$.

\subsection{Minimization interval for reservoir curve fitting}

The idea is to fit the reservoir curve (8) to the measured one such that $p_{\text {res }}$ closely aligns $p$ when wave activity is believed to be minimal. It is common to consider approximately the last two thirds of diastole for this purpose $[6,15]$, i.e. $T_{s}+\left(T_{b}-T_{s}\right) / 3 \leq t \leq T_{b}$.
However, in literature one may also find a fitting over the whole diastole [12]. Therefore, both intervals will be regarded in the comparison.

\subsection{Implementation of the RWP}

The implementation of the RWP can be done in several ways. The one presented in here basically follows the explanations in [15]:

1. Determine the duration $T_{b}$ of the given heart beat.

2. Estimate the duration of systole $T_{S}$ by means of (9) or (10).

3. Fit the reservoir pressure to the measured curve by using the assumed exponential decay (8) of $p_{\text {res }}$ during diastole. Therefore, find $b, p_{\text {res }}\left(T_{*}\right), P_{\infty}>0$ such that

$$
\left\|p_{\text {res }}-p\right\|_{\ell^{2}\left(T_{*}, T_{b}\right)} \rightarrow \min
$$

whereby $T_{*}$ denotes the start of the minimization interval in diastole. Thus, $T_{*}$ is meant to correspond to either $T_{s}$ or $T_{s}+\left(T_{b}-T_{s}\right) / 3$ associated with the respective systolic durations of (9) and (10). The minimization (11) is realized via the MATLAB function lsqnonlin. The initial parameters for the optimization are set to

$$
b_{0}:=3 \cdot 1 / \mathrm{s}, \quad p_{\text {res }}\left(T_{*}\right)_{0}:=p\left(T_{*}\right), P_{\infty, 0}:=\min p
$$

with the constraint that $30 \mathrm{mmHg} \leq P_{\infty} \leq P_{\infty, 0}$.

4. The remaining rate constant $a$ is determined by seeking continuity at $T_{*}$ between the piecewisely defined reservoir curve via (7) in systole and (8) in diastole. Hence, find $a>0$ such that their absolute difference is minimal at the transition time $t=T_{*}$. In MATLAB the optimization is done by using again lsqnonlin with initial value $a_{0}:=15 \cdot 1 / \mathrm{s}$, whereby the integral in (7) is implemented via the function trapz.

\section{Results}

In this section the obtained pressure separation according to the algorithm above is illustrated. Moreover, the effects of the choice of estimated systolic duration and minimization interval on the RWP results are presented.

\subsection{Separation of pressure}

In Figure 1 the pressure separation based on the estimated systolic duration $T_{s, d}$ and the use of the whole diastole for reservoir curve fitting is shown. 
For illustration purposes the diastolic blood pressure (DBP), defined as the minimum of the waveform $p$, was subtracted from the reservoir and measured pressure curves. It can be witnessed that in early systole the excess pressure $p_{e x}$ is a good approximation of the measured pressure $p$. However, after reaching its peak it declines quickly and remains approximately zero during diastole. As indicated in [6] the waveform of $p_{\text {ex }}$ is suggested to be a good approximation to the flow $q_{\text {in }}$ in the ascending aorta. In contrast, the reservoir pressure $p_{\text {res }}$ increases gradually during systole and hits its peak in late systole where it then closely aligns the measured pressure waveform.

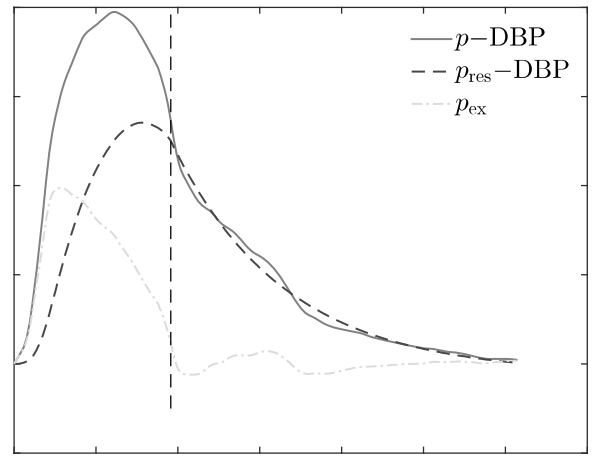

Figure 1. Pressure separation according to the RWP. For illustration purposes the DBP $=84.56 \mathrm{mmHg}$ was subtracted from both the reservoir and measured pressure waveform. The dashed vertical line indicates the estimated systolic duration $T_{s, d}=0.38 \mathrm{~s}$.

\subsection{Sensitivity on minimization interval}

In Figure 2 the reservoir curves $p_{\text {res }}(2 / 3 D, d)$ and $p_{\text {res }}(D, d)$ represent the waveforms obtained by either using two-thirds or the whole diastole associated with the estimated time $T_{s, d}$, respectively. The vertical dashdotted line indicates $t=T_{s, d}$ and the vertical dotted line the time $t=T_{s}+\left(T_{b}-T_{s}\right) / 3$, referring to the beginning of the last two-thirds of diastole. It can be observed that the later start of the optimization interval leads to a higher peak in the reservoir waveform. Since the information of the prior pressure decay in the first third is not taken into account its corresponding reservoir pressure is only determined by a small range of $p$-values at later diastole. In contrast, the reservoir waveform $p_{\text {res }}(D, d)$ is computed by a broader range of $p$, which enables a closer matching of $p_{\text {res }}$ to the actual measured pressure decay.

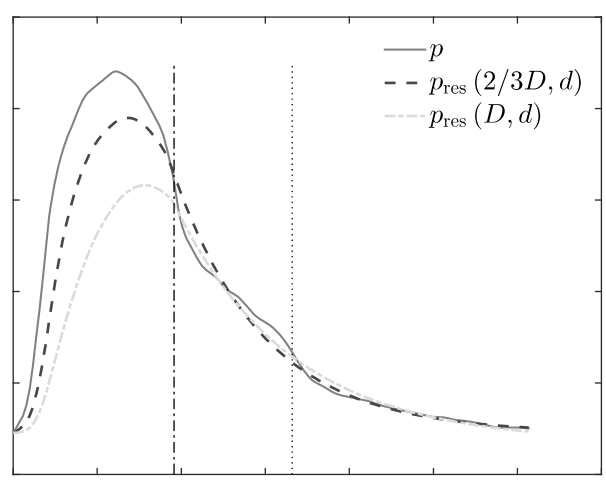

Figure 2. Reservoir pressures based on the estimation $T_{s, d}$ (vertical dash-dotted line) and its associated two-thirds of diastole $\left(p_{\text {res }}(2 / 3 D, d)\right)$ and its entire diastolic duration $\left(p_{\text {res }}(D, d)\right)$. The vertical dotted line indicates the beginning of the last two-thirds of diastole.

In Table 1 a more detailed analysis and comparison of both approaches are provided. The pulse pressure (PP) is defined as the difference between the maximum and minimum pressure value. The pressure area (A) is computed as the integral of the respective pressure curve subtracted by the DBP. By taking the reservoir waveform $p_{\text {res }}(D, d)$ as the reference the relative differences of about $27 \%$ in PP and almost $22 \%$ in area quantify the already witnessed discrepancies. The figures of both asymptotic pressures $P_{\infty}$ are close to the upper limit of the DBP $=84.56 \mathrm{mmHg}$ as a consequence of the smooth decay of $p$ at the end of diastole.

\begin{tabular}{ccccc}
\hline & 2/3D,d & D,d & abs, diff. & rel. diff. [\%] \\
\hline PP [mmHg] & 34.42 & 27.06 & 7.37 & 27.22 \\
\hline A [mmHg s] & 15.10 & 12.42 & 2.68 & 21.58 \\
\hline $\boldsymbol{P}_{\infty}$ [mmHg] & 84.56 & 83.46 & 1.09 & 1.31 \\
\hline \multicolumn{4}{c}{ Table 1. Overview of the pulse pressures (PP), areas (A) } \\
and asymptotic pressures $P_{\infty}$ of the waveforms \\
$p_{\text {res }}(2 / 3 D, d)$ and $p_{\text {res }}(D, d)$, respectively.
\end{tabular}

Based on the computed reservoir pressures of the respective minimization intervals, the results of the corresponding excess pressures $p_{\text {ex }}(2 / 3 D, d)$ and $p_{\text {ex }}(D, d)$ are presented in Figure 3 . Since generally $p_{\text {res }}(2 /$ $3 D, d) \geq p_{\text {res }}(D, d)$ during systole, it holds that $p_{\text {ex }}(2 / 3 D, d) \leq p_{\text {ex }}(D, d)$ in this period of time according to (1). 


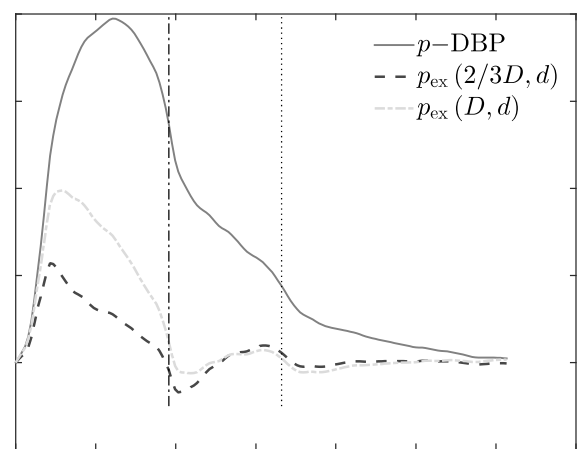

Figure 3. Excess pressures based on the estimation $T_{s, d}$ (vertical dash-dotted line) and its associated two-thirds of diastole $\left(p_{e x}(2 / 3 D, d)\right)$ and its entire diastolic duration $\left(p_{e x}(D, d)\right)$. The vertical dotted line indicates the beginning of the last two-thirds of diastole.

\subsection{Sensitivity on estimation of systolic duration}

Figure 4 shows the respective reservoir waveforms obtained by minimizing the differences of (8) and the measured waveform $p$ in entire diastole associated with both estimations $T_{s, c}$ and $T_{s, d}$. It holds that $T_{s, c}>T_{s, d}$ and that the pulse pressure of $p_{\text {res }}(D, d)$ is slightly higher during systole but no substantial differences in reservoir waveforms are observable.

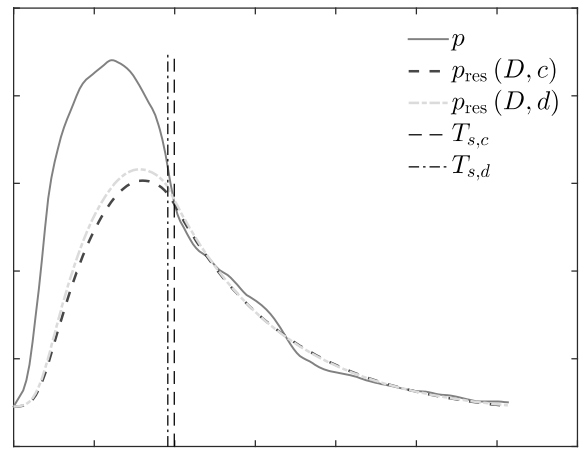

Figure 4. Reservoir pressures based on the minimization along entire diastole based on different estimations of systolic duration $T_{s, c}$ $\left(p_{\text {res }}(D, c)\right)$ and $T_{s, d}\left(p_{\text {res }}(D, d)\right)$.

Table 2 enables a more detailed discussion of the differences: The estimated systolic duration $T_{s, c}$ based on the maximum curvature of $p$ is approximately $0.02 \mathrm{~s}$ longer compared to its counterpart $T_{s, d}$ which relies on the minimum derivative.
Furthermore, only slight discrepancies are visible in terms of pulse pressure and area. The reservoir waveforms related to $T_{s, c}$ are about $-5 \%$ and approximately $4 \%$ smaller in pulse pressure and in area than their $T_{s, d^{-}}$ counterparts. Additionally, only minor differences are noticeable between their asymptotic pressures too whereby the longer time $T_{S, c}$ yields a slightly lower asymptotic pressure.

\begin{tabular}{lcccc}
\hline & $T_{s, c}$ & $T_{s, d}$ & abs. diff. & rel. diff. [\%] \\
\hline $\boldsymbol{T}_{s}$ [s] & 0.40 & 0.38 & 0.02 & 4.08 \\
\hline PP [mmHg] & 25.78 & 27.06 & -1.28 & -4.72 \\
\hline A [mmHg s] & 11.98 & 12.42 & -0.44 & -3.57 \\
\hline $\boldsymbol{P}_{\infty}[\mathrm{mmHg}]$ & 83.22 & 83.46 & -0.24 & -0.29 \\
\hline
\end{tabular}

Table 2. Overview of the pulse pressures (PP), areas (A) and asymptotic pressures $P_{\infty}$ of the waveforms $p_{\text {res }}(D, c)$ and $p_{\text {res }}(D, d)$, respectively.

\section{Discussion}

In this work, the concept of the RWP as well as a possible implementation and its sensitivity to the numerical realization were presented. The theoretical concept of pressure separation according to the RWP might seem natural due to the mentioned phenomena of aortic compliance and wave propagation, which can also be seen in the results shown in Figure 1. The reservoir pressure represents the cushioning and recoiling effect which smooths the pulsatile pressure and flow of every cardiac ejection: During the ejection period the left ventricle pumps blood into the arterial system where the 'blood storage' gets filled steadily. After closure of the aortic valves the ascending aorta forwards the blood and, thus, enables a perfusion at tissue level throughout the cardiac cycle.

Nevertheless, a concise definition of the application of this concept onto a pressure waveform is difficult to state. Aguado-Sierra et al. [15] for example use approximately the last two thirds of diastole to fit the reservoir pressure (8) to the actual pressure waveform. Parker et al. [12] use (10) as $T_{s}$-estimator and fit the assumed exponential decay of the reservoir pressure during the whole diastole. Thereafter, they choose the first crossover point of the resulting curves (7) and (8) as transition to define the entire reservoir curve. 
Additionally, the MATLAB function fminsearch is taken for the minimization itself. Vermeersch et al. [16] primarily use the method as described in [12], but switch to the last two thirds for curve fitting when the procedure is not successful for entire diastole. Moreover, they assume the asymptotic pressure $P_{\infty}$ to be zero.

Apart from that, the optimization criteria could be adapted. Other norms as the $\ell^{2}$-norm used in here are possible and different parameter ranges may be permitted, including different initial values and boundary conditions [17].

Consequently, one may find different suggestions of the RWP implementation in literature which, in turn, are based on the set of available test curves. The results presented in this work indicate a substantial impact of the minimization interval on the reservoir pressure waveform. The reservoir curves related to the two regarded different optimization intervals show remarkable differences of almost $30 \%$ in their corresponding reservoir pulse pressures. In contrast, the differences caused in asymptotic pressures may be considered as negligible. In this context, one should bear in mind that the obtained figures depend strongly on the underlying exemplary pressure curve and might differ markedly to other ones.

In conclusion, whereas the actual discussion regarding scrutiny of the RWP mainly focuses on its justification related to physiological aspects, the results presented in this work also suggest a considerable sensitivity associated with the numerical and algorithmic implementation. Consequently, a broad consensus about the algorithms used is needed before the RWP can be incorporated into clinical practice.

\section{References}

[1] Mancia G, De Backer G, Dominiczak A, et al. Guidelines for the management of arterial hypertension. Eur Heart J. 2007; 28: 1462-536.

[2] Laurent S, Cockcroft J, Van Bortel L, Boutouyrie P, Giannattasio C, Hayoz D, Pannier B, Vlachopoulos C, Wilkinson I, Struijker-Boudier H. Expert consensus document on arterial stiffness: methodological issues and clinical applications. Eur Heart J. 2006; 27(21): 2588605.

[3] Hametner B, Wassertheurer S, Hughes A, Parker K, Weber T, Eber B. Reservoir and excess pressures predict cardiovascular events in high-risk patients. Int J Cardiol. 2014; 171: 31-6.
[4] Weber T, Wassertheurer S, Rammer M, Haiden A, Hametner B, Eber B. Wave Reflections, Assessed With a Novel Method for Pulse Wave Separation, Are Associated With End-Organ Damage and Clinical Outcomes. Hypertension. 2012; 60: 534-41.

[5] Weber T, Wassertheurer S, Hametner B, Parragh S, Eber B. Noninvasive methods to assess pulse wave velocity: comparison with the invasive gold standard and relationship with organ damage. J Hypertens. 2015; in press. http://dx.doi.org/10.1097/HJH.0000000000000518.

[6] Wang JJ, O'Brien AB, Shrive NG, Parker KH, Tyberg JV. Time-domain representation of ventricular-arterial coupling as a windkessel and wave system. Am J Physiol Heart Circ. 2003; 284(4): H1358-68.

[7] Westerhof N, Westerhof BE. The reservoir wave paradigm discussion. J Hypertens. 2015; 33(3): 458-60.

[8] Mynard JP, Smolich JJ, Avolio A. The ebbing tide of the reservoir-wave model. J Hypertens. 2015; 33(3): 461-4.

[9] Davies JE, Hadjiloizou N, Leibovich D, Malaweera A, Alastruey-Arimon J, Whinnett ZI, Manisty CH, Francis DP, Aguado-Sierra J, Foale RA, Malik IS, Parker KH, Mayet J, Hughes AD. Importance of the aortic reservoir in determining the shape of the arterial pressure waveform - The forgotten lessons of Frank. Artery Res. 2007; 1(2): 40-5.

[10] Nichols W, O'Rourke M, Vlachopoulos C. McDonald's blood flow in arteries. London: Hodder Arnold; 2011. $6^{\text {th }}$ ed.

[11] Westerhof N, Lankhaar J, Westerhof B. The arterial Windkessel. Med Biol Eng Comput. 2009; 47(2): 131-41.

[12] Parker KH, Alastruey J, Stan GB. Arterial reservoirexcess pressure and ventricular work. Med Biol Eng Comput. 2012; 50(4): 419-24.

[13] Johnson DA, Spaeth JR, Rose WC, Naik UP, Beris AN. An impedance model for blood flow in the human arterial system, Part I: Model development and MATLAB implementation. Comput Chem Eng. 2011; 35(7): 1304-16.

[14] Olufsen MS. Modeling flow and pressure in the systemic arteries. In: Ottesen JT, Olufsen MS, Larsen JK, editors. Applied Mathematical Models in Human Physiology. Philadelphia: SIAM; 2004.

[15] Aguado-Sierra J, Alastruey J, Wang JJ, Hadjiloizou N, Davies J, Parker KH. Separation of the reservoir and wave pressure and velocity from measurements at an arbitrary location in arteries. Proc Inst Mech Eng H. 2008; 222(4): 403-16.

[16] Vermeersch SJ, Rietzschel ER, De Buyzere ML, Van Bortel LM, Gillebert TC, Verdonck PR, Segers P. The reservoir pressure concept: The 3-element windkessel model revisited? Application to the Asklepios population study. J Eng Math, 2009; 64: 417-28.

[17] Ebner M. Reservoir Theory and its Application on Peripheral Arteries [Master thesis]. [Institute for Analysis and Scientific Computing, (AT)], Vienna University of Technology; 2014. 